Classification

Physics Abstracts

82.70

\title{
Polymer microdises
}

\author{
N. A. Clark (*) \\ CNRS, Centre de Recherche Paul Pascal, Domaine Universitaire, 33401 Talence, France
}

(Reçu le 24 septembre 1984, révisé le 16 janvier 1985, accepté le 25 janvier 1985)

\begin{abstract}
Résumé. - On peut fabriquer des microdisques en déformant de façon plastique des microsphères polymériques. Ces disques sont biréfringents; de plus, ils se comportent comme des "vides diélectriques ", s'orientant parallèlement à un champ électrique $\mathbf{E}$, en formant des chaînes le long des lignes de champ.

Abstract. - Microdiscs can be formed by plastic deformation of polymer microspheres. These discs are birefringent, and behave as "dielectric holes", orienting parallel to an applied electric field $\mathbf{E}$ and forming chains along $\mathbf{E}$.
\end{abstract}

Spherical polymer particles can now be made with a wide variety of chemical properties and are commercially available in diameters ranging from about $0.04 \mu \mathrm{m}$ to about $10 \mu \mathrm{m}$ [1]. These particles have proved to be particularly useful in the physical and chemical study of the single particle and collective behaviour of colloidal suspensions [2,3]. By contrast, the selection of nonspherical particles for similar studies where particle shape and orientation may be important is much more limited. There are, for example, rod-shaped tobacco mosaic virus [4], prolate ellipsoidal shaped shear-fixed erythrocytes [5], disc-shaped nonshear fixed erythrocytes and bentonite crystals. In this Letter we describe a technique whereby disc-shaped polymer particles of micron dimensions can be made. This technique consists of plastic deformation of initially spherical polymer particles. Hence the wide availability of an experience with spherical polymer particles can be usefully exploited.

The fabrication of disc-shaped particles is achieved by placing a suspension of spherical particles in a specially constructed cell in the intense sound field of an ultrasonic agitator. Results described here were obtained with aqueous suspensions of 2.1 micron diameter polystyrene spheres. The cell is shown in figure 1 . It consists of a pair of standard $1 \mathrm{~mm}$ thick glass microscope slides made into a closed cell by epoxy around the edge. The spacing between the slides was established by a pair of quartz fibers or metal wires of diameter between $30 \mu \mathrm{m}$ and $100 \mu \mathrm{m}$, one near the cell centre and one at the edge, both running the length of the cell. To make the cell the slides were clamped together along the edges indicated by the arrows in figure 1 . This ensured good contact between the centre fiber and the plates. These two edges were then epoxied while clamped and

(*) Permanent Address : Department of Physics, Condensed Matter Laboratory, University of Colorado, Boulder, Colorado 80309, U.S.A. 


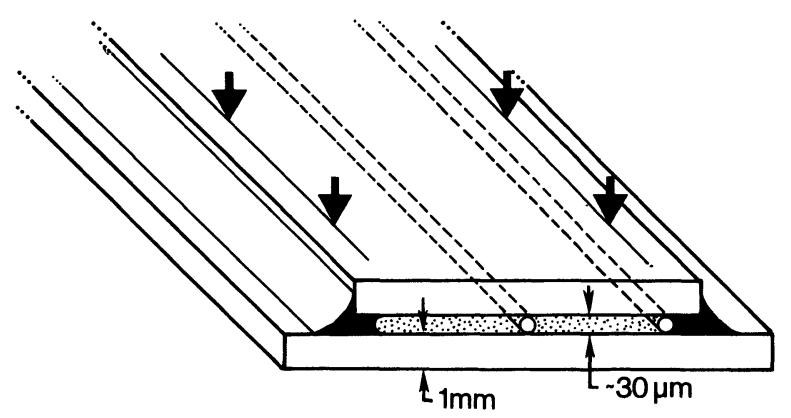

Fig. 1. - Schematic of the cell geometry for fabrication of microdiscs (not to scale). The central fiber, clamped between the plates and immersed in the sphere suspension, is essential to the disc making process, which is activated by immersion of this cell into an ultrasonic bath.

the cell was filled with suspension by capillary suction from one of the open ends. The slides were previously cleaned in hot laboratory detergent solution and hot chromic acid and rinsed in filtered reagent grade (Type I) distilled water to assure uniform capillary filling and to leave the slides dust free, giving good contact with the cylinder. Once filled the cell was sealed by epoxy on the remaining two ends and the clamps removed upon curing. The suspensions used were dilute, between 0.1 and 0.01 weight percent.

In order to convert spheres to discs the cell was immersed in an aqueous sonication bath provided by a standard ultrasonic cleaner for a period of 1 to 3 minutes. Figure 2 shows the results of this treatment. Figure $2 \mathrm{a}$ is a micrograph of the original suspension, showing the $2.1 \mu \mathrm{m}$ diameter spherical polystyrene particles. Figure $2 b$ shows typical results of the sonication treatment. Nearly all of the particles have been rendered anisotropic, with a significant number of them forming perfect discs of diameter 4 to $5 \mu \mathrm{m}$. Particle volume conservation yields disc thicknesses $d \sim 0.3 \mu \mathrm{m}$, which is consistent with the discs appearing as thin lines when viewed on edge (Fig. 2).

a)

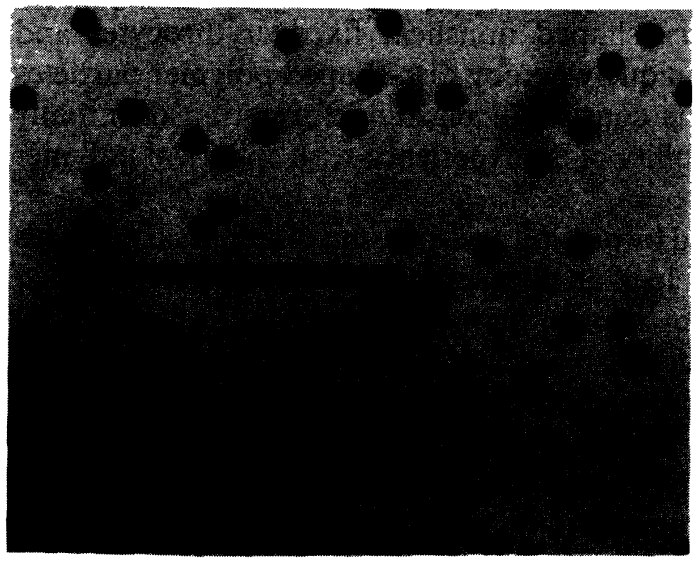

b)

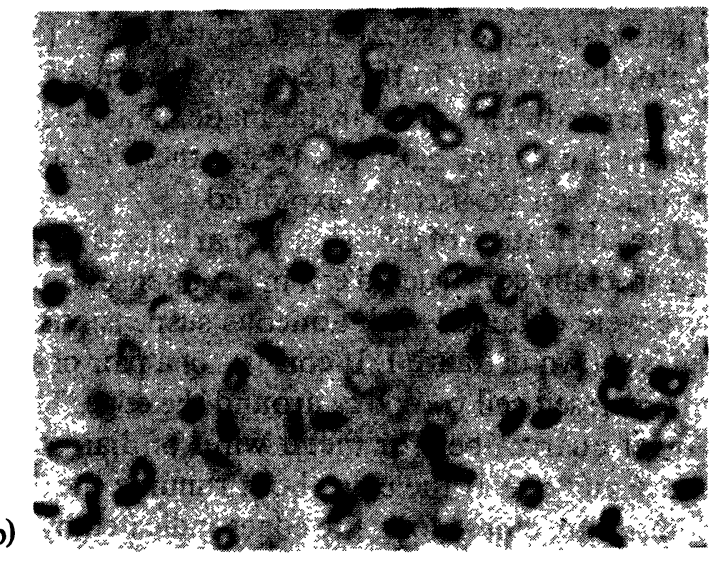

Fig. 2. - Transmission photomicrographs of spheres and discs obtained using a Leitz Diavert microscope. The bar is $50 \mu \mathrm{m}$ long. (a) Starting microspheres (diameter $2.1 \mu \mathrm{m}$ ) in aqueous suspension. (b) Discs produced by three minutes of sonication in the cell of figure 1 . 
Several features of the cell construction are essential for the formation of discs. First, sonication in cells having no central fiber had no observable effect on the shape of the starting spheres. The cylinder-plate contact, immersed in the particle suspension appears to be essential to the disc making process. On the other hand sonication in cells having many fibers was not significantly more efficient than that for a single fiber. A consequence of introducing many fibers is that because of variations in plate flatness and fiber diameter the fibers in general do not contact the plates, and in fact may be many microns away. Hence, good contact between the fibers and plates appears to be important. Of the fiber materials tested, quartz produced the best results, with the disadvantage of the fibers sometimes breaking under sonication. Enameled copper wire also worked well, but sonication left enamel particles in the disc suspension. On the other hand, copper wire etched in chromic acid solution to remove the enamel produced flocculation of the particles in the sound field, perhaps by cavitation, yielding large clumps, but no discs.

These observations lead to the following proposed model for the disc making process, illustrated in figure 3 . The sonication field produces oscillatory pressure variations which periodically open a gap between the cylinder and plate, drawing in suspension (Fig. 3b). In addition there is a local flow past the cylinder accompanying the general circulation caused by nonlinear effects in the intense sound field in the bath. In the gap between the cylinder and plate these processes combine to produce a low Reynolds number flow which is characterized by regions of zero lateral velocity (normal to the cylinder axis and parallel to the plate). Particles sufficiently close to these regions will not have sufficient lateral velocity to escape as the cylinder and plate come back into contact in the pressure cycle. These trapped particles are plastically deformed, probably in a single such cycle, returning to suspension as the gap opens on the next cycle. There is some direct evidence for this process in that, upon microscopic observation of cells which were used to make discs, many particles are found to be trapped directly between the quartz fiber and slide. The forces available are more than adequate to produce plastic deformation of the particles. Assuming a (high) yield stress for polystyrene of $10^{10}$ dynes $/ \mathrm{cm}^{2}$, the force required to flatten a $1 \mu \mathrm{m}$ diameter sphere is $\sim 1000$ dynes. Hence the force of the $\sim 100 \mathrm{~g}$ clamping pressure on the top slide could flatten $\sim 100$ spheres.

a
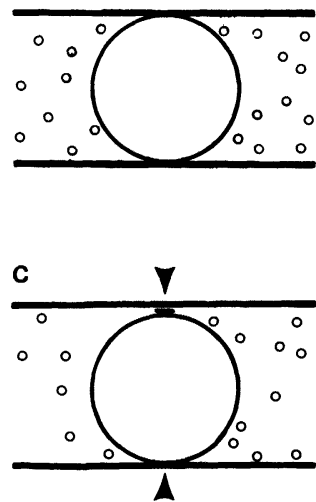

b
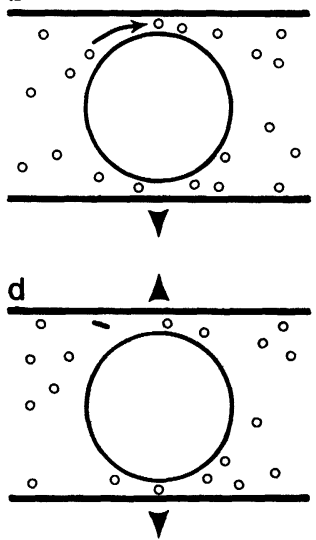

Fig. 3. - Proposed process of disc fabrication. The alternating pressure field of the sonication bath separates the central cylinder from the bounding plates, drawing in suspension. The overall (incompressible) flow pattern can leave particles in position where they are trapped and flattened during the compression cycle. 
A further interesting feature of this fabrication process is that it leaves the discs optically anisotropic. The discs behave as small birefringent plates, depolarizing the transmitted light. Consider a plane electromagnetic wave of intensity $I_{0}$ and wave vector $\mathbf{k}$ polarized along $\hat{\mathbf{e}}$, and incident on a disc in the suspension having an axis parallel to a unit vector $\hat{a}$. If $\theta$ is the angle between $\hat{\mathbf{k}}$ and $\hat{\mathbf{a}}$ and $\psi$ the angle between $\hat{\mathbf{e}}$ and the projection of $\hat{\mathbf{e}}$ normal to $\hat{\mathbf{k}}$, the transmission coefficient of an individual disc will be $T=I / I_{0}=\sin 2 \psi \sin ^{2} \phi$ where $\phi=2 \pi t / \lambda_{\mathrm{v}}\left[n_{\mathrm{o}} d_{\mathrm{o}}(\theta)-n_{\mathrm{e}}(\theta) d_{\mathrm{e}}(\theta)\right]$. Here $\lambda_{\mathrm{v}}$ is the vacuum wavelength, $n_{\mathrm{o}}, n_{\mathrm{e}}, d_{\mathrm{o}}$ and $d_{\mathrm{e}}$ are the ordinary and extraordinary refractive indices and propagation distances, respectively. For parameters of relevance here $(d \sim 0.3 \mu \mathrm{m}$, $n_{\mathrm{o}}-n_{\mathrm{e}} \sim 0.1$, visible light experiences a maximum birefringent phase shift of $\sim 0.2 \pi$ radians. Neglecting diffraction effects, i.e., for $\theta \lesssim 60^{\circ}$ the power transmitted by an individual disc will be $P=I t \pi r^{2} \cos \theta$. For $r=2 \mu \mathrm{m}$ and typical microscope or laser illumination $\left(\sim 1 \mathrm{~W} / \mathrm{cm}^{2}\right)$, an individual disc can transmit up to $\sim 10^{4}$ photons/s. Hence individual discs should be visible in transmission between crossed polarizer and analyser, and indeed they are, as shown in figure 4 . Observation of single discs qualitatively confirms the dependence of $T$ and $\psi$ and $\theta$. Furthermore, since the characteristic structural diffusion reorientation time $\left(\sim 8 \pi \eta r^{3} / k T\right)$ a $2 \mu$ disc in water is $\sim 1 \mathrm{~s}$, there are many photons per reorientation and the reorientation dynamics may be effectively studied using photon correlation spectroscopy. These experiments are underway.

Next let us consider the behaviour of these discs in an applied a.c. electric field. The disc material, being polystyrene, has a dielectric constant of $\varepsilon_{\mathrm{d}} \sim 3$, much less than that of the surrounding water, $\varepsilon_{\mathrm{w}} \sim 80$. Hence the discs behave as " dielectric holes ", acquiring field induced electric dipoles opposite the applied field. In addition, the discs experience field induced torques. Assuming the discs to be thin compared to their diameter, and ignoring the dielectric anisotropy of the disc material (permissible since $\varepsilon_{\mathrm{w}} \gg \varepsilon_{\mathrm{d}}$ ), it is easy to show that the orientation having the disc axis normal to the applied field has the lowest electrostatic energy. Hence single discs in a high field exhibit orientational Brownian motion in a single angular variable - that giving the orientation about the applied field direction. In more concentrated suspensions the induced dipoles interact to produce chaining of the discs, as shown in figure 5, similar to that observed for biological cells [6] and recently for spherical " magnetic holes » in an applied magnetic field [7]. Reduction of the electric energy density in the water is affected by adjacent discs in the chain orienting

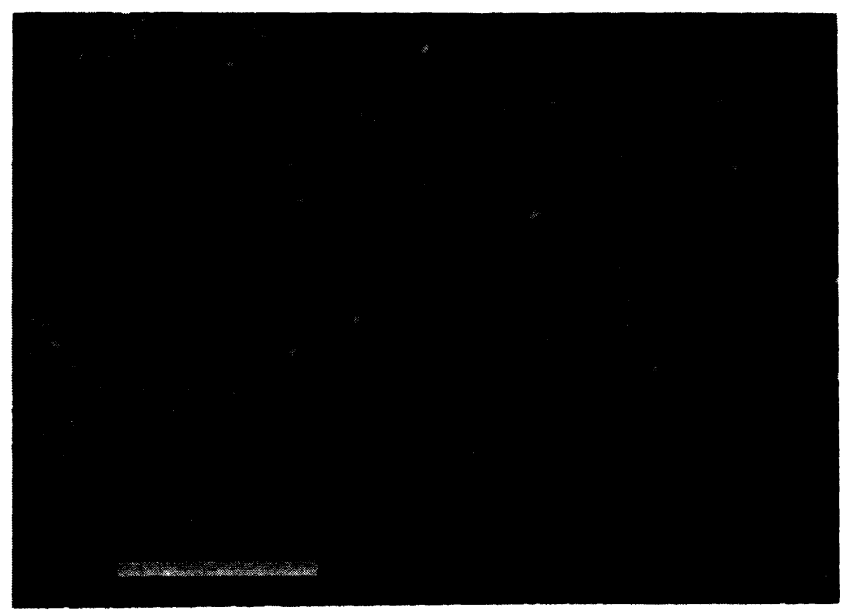

Fig. 4. - Transmission photomicrograph of a suspension of discs between crossed polarizers. The optical anisotropy of the discs causes depolarization of transmitted light for discs not having a symmetry axis projection along or normal to the polarizer direction. The initial sphere suspension is completely extinguished under similar conditions. The white bar is $300 \mu \mathrm{m}$ long. 


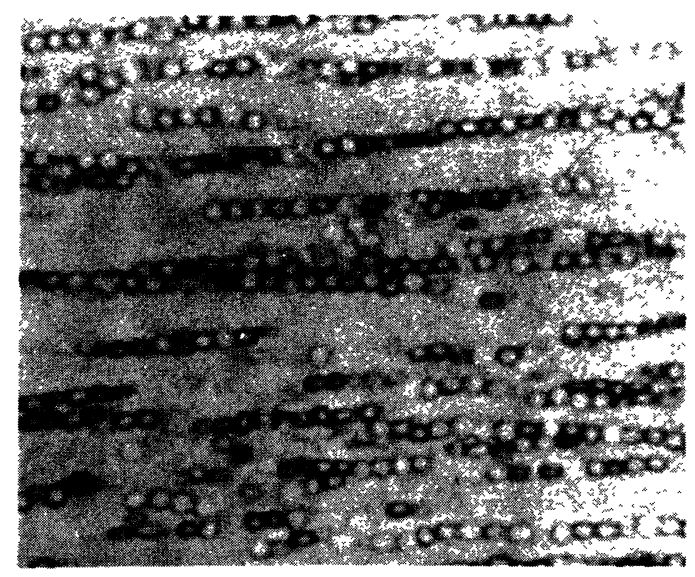

Fig. 5. - Chaining of discs induced by an applied electric field. The individual discs orient to minimize the electrostatic interaction with their axes normal to the field and form chains parallel to the field because of the induced electric dipole moments. Neighbouring discs in the chains orient parallel to reduce the field energy in the water. Scale as in figure 2.

parallel to each other. Hence a typical configuration has the azimuthal orientation of discs about the chain axis changing only slowly as one proceeds along the chain, i.e., this orientation now exhibits collective behaviour.

There are several evident extensions and applications of these observations. First, the process as described does not produce suspensions of identical discs. Clearly some particles may never be trapped while other may be compressed several times. Since for many applications monodisperse suspensions would be desirable, ways of obtaining unique shapes need to be developed. These might involve the centrifugal fractionation or laser cell sorting techniques applied to biological systems. An alternative possibility would be to make single pass cells, flowing the suspension past the cylinder at a rate that would ensure at most a single encounter per particle. One would then obtain a bimodal distribution of spherical and deformed particles which could be readily separated centrifugally. One possible application of this work concerns the study of rotational Brownian motion or the motion of anisotropic particles in flows, as it is possible in the microscope or in transmitted depolarized light to follow the detailed orientational trajectory of individual discs. Finally, this method may be of use in the rupturing of biological cells when only small samples are available.

\section{Acknowledgments.}

The author acknowledges the support of CNRS and NSF Grant DMR 82-06472, and the assistance of the members of the CRPP liquid crystal group, especially J. Prost.

\section{References}

[1] See, for example, Duke Scientific C orp., Palo Alto, California 94306, U.S.A.

[2] Pieranski, P., Contemp. Phys. 24 (1983) 25.

[3] Fitch, R. M., Polymer Colloids, 1971 ; Polymer Colloids II, 1980 (Plenum, New York).

[4] Oster, G., J. Gen. Physiol. 33 (1950) 445.

[5] Sutera, S. P. and Boylan, C. W., J. Colloid Interface Sci. 73 (1980) 295.

[6] PoHL, H. A., Dielectrophoresis : The Behavior of Matter in Nonuniform Electric Fields (Cambridge Univ. Press : London) 1978.

[7] SkJeltorp, A. T., Phys. Rev. Lett. 51 (1983) 2306. 